\title{
Entre la présence et l'intégration sociale : les modes de vie des personnes recevant des services de suivi intensif en équipe dans la communauté
}

\author{
Christiane Bergeron-Leclerc \\ Université du Québec à Chicoutimi \\ Bernadette Dallaire \\ Université Laval
}

\begin{abstract}
RÉSUMÉ
Quarante ans après l'amorce du processus de désinstitutionalisation, l'intégration sociale des personnes ayant des troubles mentaux graves constitue toujours un enjeu. De fait, leurs conditions de vie sont davantage marquées par l'exclusion, tant d'un point de vue social qu'économique. Afin de faire face à cette situation, et soutenir les personnes dans leur cheminement, a été développé le suivi intensif en équipe dans la communauté. Alors que jusqu'à présent, le regard porté sur ce programme a été surtout évaluatif, cette étude qualitative exploratoire a été menée dans l'optique d'identifier les facteurs qui facilitent ou nuisent au cheminement vers l'intégration sociale de ces personnes. Les études de dossiers et des entrevues semi-dirigées réalisées auprès des 9 participants et participantes de l'étude révèlent l'existence d'une diversité de parcours de vie communautaire. En effet, les analyses de données verticales et transversales ont permis de mettre en évidence 3 modes de vie (nomade, domestique et social), reflétant les façons d'interagir et de participer de ce groupe d'individus. Ces résultats contribuent à lever le voile sur différents enjeux associés à la pratique du suivi intensif en équipe dans la communauté.
\end{abstract}

Mots clés : Intégration sociale, exclusion sociale, modes de vie, vie communautaire, troubles mentaux graves, schizophrénie, suivi intensif en équipe dans la communauté

Christiane Bergeron-Leclerc, Ph.D., Département des Sciences humaines, Université du Québec à Chicoutimi ; Bernadette Dallaire, Ph.D., École de Service social, Université Laval.

Cet article découle d'une étude doctorale menée par l'auteure principale ; il est incorporé dans une version différente dans la thèse de l'étudiante concernée. Cette étude s'est déroulée dans le respect des normes de recherche réalisées à l'endroit de sujets humains (certification émise par le Comité d'éthique et de la recherche du Centre de recherche Université Laval Robert-Giffard : numéro 121).

La correspondance concernant cet article devrait être acheminée à Christiane Bergeron-Leclerc, Ph.D., professeure régulière, Département des Sciences Humaines, Université du Québec à Chicoutimi, 555, Boulevard Université, Chicoutimi (Québec), G7H 2B1. Téléphone : 418-545-5011 poste 4230. Télécopieur : 418-545-5012. Courriel : christiane_bergeron-leclerc@uqac.ca 
Au cœur du processus de désinstitutionalisation qui s'est amorcé dans les années 1960 existait une idée maîtresse : celle d'une plus grande intégration sociale des personnes ayant des troubles mentaux. Alors que pour les personnes ayant des affections légères ou modérées le rêve s'est concrétisé, pour le groupe d'individus ayant les troubles les plus graves, la réalité s'est avérée beaucoup plus sombre (Gélinas, 2002 ; Guy, 1997). Ainsi, plusieurs de ces personnes vivent dans un logement qu'elles considèrent insatisfaisant, ont peu d'activités significatives et se sentent seules et isolées socialement, en plus de vivre dans des conditions économiques précaires (Bradshaw, Armour, \& Roseborough, 2007 ; Davidson, Hoge, Godleski, Rakfeldt, \& Griffith, 1996). Bref, leurs conditions de vie sont marquées par l'exclusion plutôt que par l'intégration sociale.

Pourtant, depuis les années 1970, plusieurs programmes destinés à favoriser le parcours d'intégration ont été mis sur pied. C'est notamment le cas du suivi intensif en équipe dans la communauté (ACT ${ }^{1}$ ), programme abondamment étudié, et qui en dépit des effets observés au plan clinique et psychosocial est surtout reconnu pour son effet en termes de réduction de la durée des hospitalisations (Drake, 1998 ; Latimer, 1999 ; Stein \& Test, 1980). Alors que jusqu'à présent le regard porté sur le modèle ACT a été surtout évaluatif, l'étude dont il est ici question ici vise à mieux comprendre l'expérience des personnes intégrées à ce type de programme. Plus spécifiquement, notre objectif était d'identifier les facteurs qui facilitent ou nuisent aux cheminements d'intégration sociale des personnes suivies par une équipe ACT québécoise, soit celle de l'Institut universitaire en santé mentale de Québec. Le présent article se centre sur un volet de nos résultats, à savoir la description des modes de vie communautaire des personnes intégrées à ce programme. Après un survol des écrits, les résultats sont présentés, puis discutés à la lumière de certains enjeux pour la pratique du suivi intensif en équipe dans la communauté.

\section{L'INTÉGRATION SOCIALE DES PERSONNES AYANT DES TROUBLES MENTAUX GRAVES}

Bien que le concept ait initialement été défini en termes de «maintien » dans la communauté, on sait maintenant que la simple présence physique, tout en étant une condition nécessaire, n'est pas suffisante pour que l'on puisse parler d'intégration sociale (Aviram \& Segal, 1977 ; Rosenblatt \& Mayer, 1974). D'autres aspects, tels l'accroissement des liens sociaux et la participation sociale, doivent également être considérés (Granerud \& Severinsson, 2006 ; Ware, Hopper, Tugenberg, Dickey, \& Fisher, 2007). L'intégration sociale réfère donc à une plus grande capacité d'accomplissement des rôles sociaux attendus dans un contexte donné, ce qui implique, pour les personnes ayant des troubles mentaux graves (TMG), une prise de distance par rapport au système de services de santé mentale. Plus spécifiquement, Bond, Salyers, Rollins, Rapp et Zipple (2004) suggèrent qu'une personne est en voie d'intégration si : (a) elle se considère comme une personne et non pas comme un patient ou une patiente ; (b) elle habite dans un logement autonome, plutôt que dans une résidence supervisée ; (c) elle travaille dans un milieu d'emploi compétitif, plutôt que dans un atelier de travail protégé ; et (d) elle participe à des activités de loisir dans sa communauté, plutôt que dans des centres de jour en santé mentale. Cette vision de l'intégration s'apparente à ce que certains et certaines décrivent comme étant le rétablissement social : c'est-à-dire le rétablissement des conséquences associées à un trouble mental (Allott, Loganathan, \& Fulford, 2002 ; Onken, Craig, Ridgway, Ralph, \& Cook, 2007). 


\section{Entre la présence et l'intégration : des obstacles à franchir}

Encore aujourd'hui, la population des personnes ayant des TMG, largement stigmatisée, fait partie des plus exclues de la société (Leff \& Warner, 2006 ; Sayce \& Measey, 1999). Cette exclusion se fait sentir au quotidien, se traduisant notamment par l'isolement social dont souffrent ces individus (Aubry \& Myner, 1996 ; Granerud \& Severinsson, 2006 ; Roe, 2001). Il semble en effet que les personnes ayant des TMG aient moins de contacts sociaux, et que la nature de leurs relations soient davantage unidirectionnelle et non réciproque, signifiant qu'elles se retrouvent souvent dans le rôle de la personne qui reçoit, qui est aidée par autrui (Aubry \& Myner, 1996 ; Cohen \& Sokolovsky, 1978). Des recherches tendent également à démontrer que le réseau social de ces individus est essentiellement constitué de personnes utilisatrices de services et de professionnels et professionnelles de la santé mentale (Angell, 2003 ; Davidson, Haglund et al., 2001 ; Roe, 2001).

Cette réalité concernant la pauvreté des réseaux sociaux est amplifiée, d'une part, par le manque d'occupation sociale ou socioprofessionnelle et, d'autre part, par le manque de ressources financières (Davidson, Haglund et al., 2001 ; Heider et al., 2007 ; Roe, 2001). De fait, la grande majorité des personnes ayant des TMG sont sans emploi (Anthony \& Blanch, 1987). Cette difficulté à obtenir et à garder un emploi a pour effet que la plupart de ces personnes vivent de prestations d'assistance sociale, ce qui les condamne à la pauvreté et, du coup, constitue un net obstacle à leur intégration (Leff \& Warner, 2006 ; Lemaire \& Mallik, 2005 ; Nordt, Müller, Rössler, \& Lauber, 2007). Tout en affectant la satisfaction des besoins de base, de même que l'accès à un logement convenable et aux loisirs, le manque de revenu contribue à la faiblesse/ rupture des liens sociaux (Leff \& Warner, 2006). Davidson et ses collègues ont démontré que l'inverse est aussi vrai (Davidson, Haglund et al., 2001 ; Davidson, Stayner et al., 2001). Dans un projet sur le soutien à la socialisation, ils ont pu observer que l'octroi d'une allocation mensuelle de 28 \$ (USD) avait non seulement permis aux personnes ayant des TMG de s'offrir des activités qui autrement auraient été inaccessibles, mais a également favorisé la transformation des liens avec leur entourage. Le montant reçu, même modeste, a permis à ces personnes de reprendre contact avec des membres de leur famille et de s'engager dans des relations réciproques, où chaque personne donne et reçoit, et de se sortir du cycle des relations unidirectionnelles. Ainsi, à travers l'échange et le don, ils ont pu reprendre du pouvoir sur leur vie.

En somme, pour plusieurs personnes, la vie dans la communauté se résume à y être physiquement, c'està-dire à demeurer entre quatre murs et être essentiellement engagé dans des activités solitaires (Davidson, Haglund et al., 2001). Cette expérience contribue à un sentiment d'altérité par rapport à la communauté (Davidson, Haglund et al., 2001 ; Moldovan, 2007). Pourtant, et contrairement à la croyance voulant que les personnes ayant des TMG n'ont aucun intérêt pour les interactions sociales, plusieurs études indiquent que les personnes souhaitent s'impliquer socialement (Davidson et al., 2004 ; Granerud \& Severinsson, 2006). Ces personnes rêvent d'être « comme tout le monde » : ce qui peut se traduire par avoir un logement

convenable, des activités de loisirs, un emploi, des relations sociales (Anthony, 2004) et des liens amoureux (Cormier, 2009). 


\section{En route vers l'intégration : les facteurs facilitants}

Au-delà des obstacles identifiés, certains facteurs d'ordres individuel et environnemental semblent favoriser le cheminement des personnes vers un plus haut niveau d'intégration sociale. Du côté des facteurs individuels, l'âge est le déterminant ayant trouvé le plus d'appui dans les recherches, un âge moindre étant associé à un niveau accru de participation sociale (Kruzich, 1985 ; Nagy, Fisher, \& Tessler, 1988 ; van Wel, Felling, \& Persoon, 2003). En ce qui concerne le genre, le fait d'être un homme serait associé à un plus haut niveau d'intégration dans la communauté (intégration externe), alors que d'être une femme faciliterait l'intégration au sein du milieu de vie (intégration interne) (Nagy et al., 1988 ; Segal \& Everett-Dille, 1980). Les personnes ayant moins de symptômes psychiatriques et une personnalité extravertie sont également mieux intégrées (Gulcur, Tsemberis, Stefancic, \& Greenwood, 2007 ; Wieland, Rosenstock, Kelsey, Ganguli, \& Wisniewski, 2007). Certains travaux indiquent aussi que les personnes qui possèdent davantage d'habiletés sociales, ou qui ont un meilleur niveau de fonctionnement social, s'intègrent mieux (Kennedy, 1989; Kruzich, 1985 ; Prince \& Prince, 2002).

Du côté des facteurs environnementaux, si l'isolement est un obstacle, la présence d'un réseau de soutien facilite le cheminement vers l'intégration sociale (Dailey et al., 2000 ; Kennedy, 1989 ; Prince \& Prince, 2002). À l'égard de la situation résidentielle, il semble que le fait de pouvoir choisir son logement soit positivement associé à l'intégration physique et sociale. Quant au type de logement, les études réalisées à l'égard de milieux supervisés ont fait valoir l'importance de certaines caractéristiques (ex : résidences plus petites, situées en milieu urbain, offrant un encadrement minimal) dans le cheminement des individus qui y séjournent (Kruzich, 1985 ; Nagy et al., 1988). Enfin, le fait de vivre dans une communauté de petite ou de moyenne taille (pop. 10 000-100 000) a été identifié comme favorable au cheminement vers l'intégration (Kruzich, 1985).

\section{L'intégration vue à travers le suivi intensif en équipe dans la communauté}

Bien que peu nombreuses, certaines études se sont intéressées au cheminement communautaire des personnes intégrées à des équipes de type ACT. Celles-ci ont surtout mis en valeur des composantes d'intervention susceptibles de contribuer positivement au maintien et à l'intégration sociale des personnes utilisatrices de services. Pour n'en nommer que quelques unes, l'importance de la qualité du lien thérapeutique et de la disponibilité accrue des intervenants et intervenantes, notamment en période de crise, ont été identifiés comme des éléments affectant positivement le parcours des personnes intégrées à une équipe ACT (Chinman, Allende, Bailey, Maust, \& Davidson, 1999 ; Krupa et al., 2005), rejoignant les conclusions des travaux sur les ingrédients actifs du modèle ACT (McGrew, Wilson, \& Bond, 1996 ; Prince, Demidenko, $\&$ Gerber, 2000). Si ces recherches ont l'avantage de s'intéresser aux composantes d'intervention associées à un meilleur cheminement communautaire, elles renseignent peu sur la nature du cheminement. En effet, entre le maintien dans la communauté et l'intégration sociale subsiste tout un monde, comme nous venons de le voir. C'est donc dans l'optique d'approfondir nos connaissances sur l'expérience de vie des personnes recevant des services de type ACT, notamment en ce qui a trait aux facteurs qui favorisent ou nuisent à ce passage vers un plus haut niveau d'intégration sociale, qu'a été réalisé ce projet de recherche. 


\section{MÉTHODOLOGIE}

\section{Stratégies de recrutement, de collecte et d'analyse des données}

Cette étude qualitative de type exploratoire a été réalisée en 2004-2005 auprès de 9 personnes, représentant près du quart de la population totale de l'équipe ACT de l'Institut universitaire en santé mentale de Québec (Québec) ${ }^{2}$. Ces personnes volontaires ont reçu une compensation financière de 20 \$ (CAD) en échange de leur participation. Pour être éligible à l'étude, en plus de devoir répondre aux critères d'admissibilité à l'équipe ACT (Colbert, Dubois, Fleury, \& Giguère, 2003), les participants et participantes devaient, au moment du recrutement : (a) résider dans la communauté (ne pas être hospitalisé[e]), (b) faire partie de l'équipe ACT depuis au moins 6 mois et (c) avoir passé plus de $75 \%$ de leur temps dans la communauté depuis l'intégration à l'équipe ACT. De plus, conformément au principe de variation maximale (Patton, 1990), l'échantillon a été diversifié en fonction de certains critères reconnus pour leur impact dans le processus d'intégration sociale des personnes (ex : âge de début de la maladie, durée de la maladie, abus de substances, type de milieu de vie, emploi). C'est pourquoi, en plus de la distribution de feuillets publicitaires, des approches directes auprès de personnes ayant des caractéristiques spécifiques ont été utilisées comme stratégies de recrutement.

Afin de répondre aux objectifs de recherche poursuivis, des entrevues semi-dirigées et des études de dossiers médicaux et psychosociaux ont été réalisées. Dans les deux cas, différentes facettes de la vie des personnes ont été explorées, dont : (a) l'expérience au sein de l'équipe ACT, (b) le trouble mental et ses répercussions, (c) les habitudes et occupations quotidiennes, (d) les activités sociales et socioprofessionnelles, (e) les liens sociaux, (f) la situation résidentielle et (g) les rêves et projets d'avenir. Les données issues des dossiers ont été colligées à l'aide d'une grille d'analyse et les entrevues ont été enregistrées, retranscrite sous forme de verbatim, puis analysées selon les étapes proposées par W.L. Miller et Crabtree (1999) à l'aide du logiciel NVivo (version 8.0).

En fonction des questions de recherche, une matrice à groupement conceptuel a ensuite été élaborée (Miles \& Huberman, 2003), permettant de classifier pour chacun des répondants et chacune des répondantes les informations de nature individuelle et environnementale, de même que liées à l'expérience et à la perception des participants et des participantes à l'endroit de l'équipe ACT. Cette façon d'organiser les données a facilité la réalisation des analyses verticales (intracas) et transversales (intercas) qui ont ensuite été conduites. Ces analyses ont permis de faire ressortir des similitudes et différences chez les participants et participantes. Au final, c'est sur la base des liens sociaux et de l'engagement au sein d'activités significatives, deux variables d'influence dans le cheminement vers l'intégration, qu'ont été effectués les regroupements autour des trois modes de vie communautaire ${ }^{3}$. Il importe ici de souligner que ces modes de vie constituent des tendances. En dépit des éléments qui permettent de les discriminer, et en dépit des variations observées au plan du fonctionnement et de l'intégration sociale des personnes rencontrées, celles-ci partagent plusieurs points en commun. Cela est notamment le cas en ce qui concerne l'expérience et la perception des personnes à l'égard du modèle ACT. 


\section{Profil des participants et des participantes de l'étude}

Le tableau 1 décrit les caractéristiques des 9 participants et participantes de l'étude. Les données indiquent d'abord que la majorité d'entre eux sont de sexe masculin. On constate aussi que les participants et participantes ont tous un diagnostic de psychose schizophrénique, la forme paranoïde étant la plus fréquente. En ce qui concerne le début et l'évolution de la maladie, trois quarts des personnes rencontrées ont connu un début hâtif (avant 30 ans). Considérant l'âge moyen des participants et des participantes (40,9 ans), la majorité des personnes expérimentaient des symptômes depuis plus de 10 ans. En ce qui a trait à leurs habitudes de vie, le tiers des participants et des participantes consomment de l'alcool ou des drogues à raison d'une ou de plusieurs fois par semaine. Du côté de leur situation résidentielle, près de la moitié des individus vivent de façon autonome dans un logement, tandis que le tiers habitent dans un milieu avec encadrement. Quant aux autres, ils vivent en appartement, mais avec des amis ou amies ou membres de la famille. Il est à noter que les personnes déménagent en moyenne deux fois l'an. Au point de vue financier, la très grande majorité des personnes reçoivent des prestations de la sécurité du revenu. Enfin, tous les participants masculins étaient soumis à des dispositions légales au moment de l'entrevue, les 3 femmes faisant partie de cet échantillon ne l'étant pas.

\section{Tableau 1}

Profil des participants et participantes à l'étude

\begin{tabular}{|c|c|c|c|c|c|c|c|c|}
\hline Pseudonyme* & Sexe & $\hat{A} g e$ & $\begin{array}{c}\text { Type de } \\
\text { schizophrénie }\end{array}$ & $\begin{array}{l}\text { Début/Durée } \\
\text { maladie }\end{array}$ & $\begin{array}{l}\text { Alcool/ } \\
\text { Drogues }\end{array}$ & $\begin{array}{c}\text { Situation } \\
\text { résidentielle }\end{array}$ & Revenus & $\begin{array}{c}\text { Statuts } \\
\text { légaux }^{* *}\end{array}$ \\
\hline Denis & $\mathrm{M}$ & 53 & Paranoïde & Tardif $/+$ de 10 ans & Non & $\begin{array}{l}\text { Logement/ } \\
\text { colocation }\end{array}$ & $\begin{array}{l}\text { Rentes } \\
\text { invalidité }\end{array}$ & RP : Tutelle \\
\hline Rose & $\mathrm{F}$ & 41 & Paranoïde & Hâtif/ + de 10 ans & Non & Logement/seule & Aide sociale & Aucun \\
\hline Marianne & $\mathrm{F}$ & 47 & Schizoaffectif & Hâtif/ + de 10 ans & Non & $\begin{array}{l}\text { Logement/ } \\
\text { avec famille }\end{array}$ & $\begin{array}{l}\text { Rente } \\
\text { invalidité }\end{array}$ & Aucun \\
\hline Sébastien & M & 32 & Paranoïde & Hâtif/ + de 10 ans & Non & Logement/seul & Aide sociale & $\begin{array}{l}\text { RP : Tutelle } \\
\text { TAQ }\end{array}$ \\
\hline Alain & M & 42 & Schizoaffectif & Hâtif/ + de 10 ans & Oui & Logement/seul & Aide sociale & $\begin{array}{l}\text { Autorisation } \\
\text { de soins }\end{array}$ \\
\hline Antoine & M & 27 & Paranoïde & Hâtif/ - de 10 ans & Oui & $\begin{array}{l}\text { Chambre et } \\
\text { pension }\end{array}$ & Aide sociale & RP : Tutelle \\
\hline Jérémie & M & 23 & Schizoaffectif & Hâtif/ - de 10 ans & Non & $\begin{array}{l}\text { Ressource } \\
\text { intermédiaire }\end{array}$ & Aide sociale & $\begin{array}{l}\text { Autorisation } \\
\text { de soins }\end{array}$ \\
\hline Elisabeth & $\mathrm{F}$ & 45 & Paranoïde & Tardif/ - de 10 ans & Non & Logement/seule & Aide sociale & Aucun \\
\hline François & M & 56 & Paranoïde & Hâtif/ + de 10 ans & Oui & $\begin{array}{l}\text { Chambre et } \\
\text { pension }\end{array}$ & Aide sociale & $\begin{array}{l}\text { RP : Tutelle } \\
\text { TAQ }\end{array}$ \\
\hline
\end{tabular}

*Les noms qui figurent ici sont fictifs de façon à préserver l'anonymat des participants et des participantes.

**RP : Tutelle $=$ Régime de protection de type tutelle aux biens et à la personne ; TAQ : Ordonnance du Tribunal administratif du Québec. 


\section{LES RÉSULTATS : L'IDENTIFICATION DE TROIS MODES DE VIE COMMUNAUTAIRE}

Le premier constat qui émerge de nos analyses est à l'effet que le groupe de personnes interrogées ne forme pas un tout homogène. Si elles ont en commun le fait d'avoir un trouble psychotique, d'avoir connu plusieurs épisodes d'hospitalisation et d'avoir été ciblées pour recevoir les services d'une équipe ACT, ces personnes se distinguent par leurs modes de vie, ce qui inclut leur façon d'appréhender la vie, d'occuper leur quotidien, d'interagir et de s'intégrer dans la communauté. Tenant compte de ces variations, trois tendances liées aux modes de vie des personnes ayant participé à l'étude ont été identifiées. Il s'agit des modes de vie nomade, domestique et social. Les prochaines lignes permettront de mettre en évidence les caractéristiques des individus identifiés à chacune de ces trois tendances, tout comme de leurs interactions avec leur environnement et leur façon de percevoir et de vivre leur expérience au sein de l'équipe ACT. Le tableau 2 présente une synthèse des éléments caractéristiques associés à chacun de ces trois modes de vie.

\section{Le mode nomade}

Les personnes qui ont un mode de vie nomade $(n=3)$ vivent en marge de la société. Tout en y étant, elles refusent de s'y engager outre mesure, préférant rester à l'écart et observer ce qui se passe, plutôt que d'y participer activement. Ces personnes vivent en quelque sorte un sentiment d'étrangeté face à la société et ont de la difficulté à trouver leur place et à faire leur nid dans la communauté. Est-ce le fruit : (a) de la maladie et de ses conséquences ? (b) de traits de personnalité qui rendent les interactions sociales difficiles ? (c) des effets associés à la consommation d'alcool et de drogues ? (d) des multiples allers-retours entre l'institution et la communauté ? ou (e) des épreuves de la vie et des nombreux abandons, ruptures et échecs rencontrés ? Bien qu'il soit difficile d'identifier avec précision tous les facteurs et dynamiques en cause, une chose est claire : leur tumultueux parcours de vie fait d'elles des personnes qui se disent méfiantes, qui gardent à distance, qui hésitent à s'engager socialement. Plutôt, les personnes ayant un mode de vie nomade sont en quête de liberté, d'évasion, de plaisir immédiat, fuyant en quelque sorte les responsabilités qui incombent à la vie adulte. Ces personnes ont de la difficulté à mettre en œuvre des projets ou à se projeter dans le futur, vivant plutôt au jour le jour. Leurs rêves sont davantage liés à l'acquisition de biens qu'à un réel désir d'intégration.

Ayant pratiquement toujours vécu sous le seuil de la pauvreté, ces personnes au mode de vie nomade ont appris tôt dans leur vie à se débrouiller pour survivre. Sous des airs d'insouciance se cachent cependant des personnes qui mentionnent se sentir seules et qui se disent souffrantes et insatisfaites de leur vie. Cependant, la non-reconnaissance de leurs difficultés, notamment la maladie, rend difficile l'utilisation de stratégies d'adaptation efficaces, c'est-à-dire centrées sur le problème, tout comme l'acceptation de l'aide professionnelle apportée par l'équipe ACT. L'acceptation de l'aide est d'autant plus difficile que ces personnes y ont été légalement contraintes. Ce faisant, même si elles retirent certains avantages à faire partie de l'équipe ACT et apprécient le contact avec les intervenants et intervenantes, elles se sentent brimées et réclament haut et fort leur liberté.

Autre trait caractéristique de ces personnes, elles n'aiment pas la structure ; c'est pourquoi leur quotidien est orienté autour de la réalisation d'activités libres, à l'extérieur de leur milieu de vie. Parce qu'elles ont de la difficulté à gérer leur solitude, elles aiment se retrouver parmi les autres; c'est pourquoi elles se 
Tableau 2

Caractéristiques associées aux trois modes de vie communautaire

\begin{tabular}{|c|c|c|c|}
\hline \multirow[b]{2}{*}{ Caractéristiques } & \multicolumn{3}{|c|}{ Modes de vie } \\
\hline & Nomade $(n=3)$ & Domestique $(n=3)$ & Social $(n=3)$ \\
\hline \multicolumn{4}{|l|}{ Caractéristiques individuelles } \\
\hline Genre & Hommes & Mixte (+ d'hommes) & Mixte (+ de femmes) \\
\hline Âge & $27-56$ ans & $23-47$ ans & 40 ans et + \\
\hline \multicolumn{4}{|l|}{ Maladie mentale : état de situation } \\
\hline Symptômes psychotiques & Légers/modérés & Aïgus & Aucun \\
\hline Traits ou troubles de la personnalité & Antisocial/limite & Passif & Aucun \\
\hline Anxiété & Légère/modérée & Modérée/élevée & Légère/modérée \\
\hline Abus ou dépendance aux substances & Abus ou dépendance & Aucun & Aucun \\
\hline \multicolumn{4}{|l|}{ Hospitalisation avant l'intégration à $\mathrm{ACT}^{*}$} \\
\hline Durée annuelle moyenne (jours/année) & $164,4(153,6)$ jours & $114(74,4)$ jours & 120 (48) jours \\
\hline Fréquence annuelle moyenne (fois/année) & 1,5 & 1,8 & 1,8 \\
\hline \multicolumn{4}{|l|}{ Situation résidentielle } \\
\hline Type d'encadrement dans le milieu & Supervisé/Autonome & Autonome/supervisé & Autonome \\
\hline Type de résidence & $\begin{array}{l}\text { Chambre et pension/ } \\
\text { appartement }\end{array}$ & $\begin{array}{l}\text { Appartement/chambre et } \\
\text { pension }\end{array}$ & Appartement ou maison \\
\hline Nombre annuel moyen de déménagements & 1,8 & 2,4 & 1,3 \\
\hline Structure/Routine de vie & Non & $\begin{array}{l}\text { Orientée vers les activités } \\
\text { domestiques }\end{array}$ & $\begin{array}{l}\text { Orientée vers l'engagement } \\
\text { social }\end{array}$ \\
\hline Rêves & Acquisition de biens & $\begin{array}{l}\text { Divers (ex: santé, } \\
\text { acquisition de biens) }\end{array}$ & Vie et intégration sociale \\
\hline \multicolumn{4}{|l|}{ Caractéristiques environnementales } \\
\hline \multirow[t]{3}{*}{ Composition du réseau de soutien } & Pas de contacts familiaux & $\begin{array}{l}\text { Peu ou pas de contacts } \\
\text { familiaux }\end{array}$ & Contacts familiaux réguliers \\
\hline & Pas d'ami(e)s/connaissances & $0-1$ ami(e)s/connaissances & 0-3 ami(e)s/connaissances \\
\hline & $\begin{array}{l}\text { Professionnel(le)s de } \\
\text { l'équipe ACT }\end{array}$ & $\begin{array}{l}\text { Professionnel(le)s de } \\
\text { l'équipe ACT }\end{array}$ & $\begin{array}{l}\text { Professionnel(le)s de } \\
\text { l'équipe ACT }\end{array}$ \\
\hline Type de relations sociales & Utilitaire/non réciproque & $\begin{array}{l}\text { Utilitaire/non réciproque/de } \\
\text { soutien }\end{array}$ & Réciproque/de soutien \\
\hline Participation sociale & Activités de l'équipe ACT & Activités de l'équipe ACT & $\begin{array}{l}\text { Activités sociales et } \\
\text { professionnelles }\end{array}$ \\
\hline \multicolumn{4}{|l|}{ Expérience/perceptions face à l'équipe ACT } \\
\hline Intégration à ACT par choix? & Non (ordonnance légale) & Oui/non (ordonnance légale) & Oui \\
\hline Perception à propos de l'équipe $\mathrm{ACT}$ & Négative & Négative/positive & Positive \\
\hline Élément le plus apprécié à l'égard d'ACT & $\begin{array}{l}\text { Relation avec les } \\
\text { professionnel(le)s }\end{array}$ & $\begin{array}{l}\text { Relation avec les } \\
\text { professionnel(le)s }\end{array}$ & $\begin{array}{l}\text { Relation avec les } \\
\text { professionnel(le)s }\end{array}$ \\
\hline Participation aux activités de l'équipe ACT & $\begin{array}{l}\text { Activités sportives et } \\
\text { sociales }\end{array}$ & Activités sociales & $\begin{array}{l}\text { Activités sportives et } \\
\text { sociales }\end{array}$ \\
\hline
\end{tabular}

* La durée et la fréquence annuelle moyenne ont été estimées à partir des 3 années précédant l'intégration à l'équipe ACT. Dans le cas de la durée annuelle moyenne, le nombre entre parenthèse a été calculé sur 24 mois, excluant l'année précédant l'admission à l'équipe ACT où les durées de séjour sont anormalement élevées. 
promènent dans les rues, errent dans les parcs ou encore fréquentent des lieux publics achalandés. Qui plus est, elles ont pris l'habitude de fréquenter les soupes populaires, et ce même si les repas sont fournis dans leur milieu de vie. La possibilité d'y faire des rencontres, de pouvoir échanger avec les autres, constitue l'un des avantages de ce type de ressource, comme en témoigne l'extrait qui suit :

Bien je vais à l'Auberivière. Pour voir du monde, que je connais ou me faire des nouveaux chums, je ne sais pas. On vent un peu ce qu'on a. Je m'assois. Puis je parle avec du monde que je connais. [. . .] Des fois j'y vais quand je n'ai rien à faire. Je me dis : je ne suis pas trop loin, je m'en vais aller manger là. Ça permet de voir du monde. Ça brise la solitude. Ça brise la solitude. (François)

La consommation d'alcool et de drogues fait partie intégrante du mode de vie nomade. En effet, elle occupe une place centrale, structurant en bonne partie la nature de leurs activités quotidiennes. Ainsi, afin de pouvoir se procurer les substances désirées, les personnes passent une bonne partie de leurs journées à la recherche de ressources leur permettant de financer leur consommation. Selon la période du mois et l'urgence du besoin, elles mettent en place différentes stratégies : vendre leurs biens ou les mettre en gage, échanger des services, emprunter de l'argent, mendier ou encore faire du microtrafic de stupéfiants. Toutefois la vie en maison de chambre, si elle est propice à l'instauration de ce système d'échanges et de transactions entre les résidents et résidentes, peut rapidement être compromise si la consommation d'alcool et de drogues de ces personnes interfère dans la vie de groupe. L'expulsion résidentielle constitue un enjeu important pour les personnes ayant un mode de vie nomade. En effet, sous l'effet de l'alcool et des drogues, ces personnes peuvent voir leurs symptômes psychotiques exacerbés et leurs comportements hétéroagressifs augmentés.

La consommation d'alcool et de drogues a également un impact sur la nature de leurs relations sociales. Plus spécifiquement, elle a pour effet d'instrumentaliser les relations que les personnes entretiennent avec les autres. Ces dernières ont donc tendance à se rapprocher des personnes qui peuvent être utiles à leur quête et s'éloigner des autres. N'ayant pas vraiment d'amis et d'amies et fréquentant essentiellement des personnes qui consomment ou qui sont vulnérables, elles sont régulièrement impliquées dans des relations non égalitaires. Si elles se retrouvent parfois dans le rôle de la personne qui exploite, l'inverse est aussi vrai. N'ayant plus de contacts avec leur famille, leur réseau social se limite donc à quelques connaissances de même qu'aux professionnels de l'équipe ACT, à qui, disent-elles, elles ne font pas confiance. Ce faisant, même si elles osent sortir de chez elles pour fréquenter des lieux publics et y rencontrer des gens, les personnes ayant un mode nomade n'en sont pas pour autant intégrées socialement.

\section{Le mode domestique}

Les personnes ayant un mode de vie domestique $(n=3)$ passent la grande majorité de leur temps dans leur milieu de vie. Contrairement aux personnes ayant un mode nomade, elles ont fait leur nid, mais il leur est très difficile de quitter leur environnement familier pour explorer le monde extérieur. Pour elles, anxieuses et ayant peu confiance en leurs moyens, chaque sortie dans la communauté constitue un défi, qui pour certaines

personnes devient plus facile au fil des jours. Malgré tout, elles expriment une nette préférence à vivre dans la communauté, comme le démontrent ces extraits :

C'est quelque chose [les services de l'équipe ACT] qui permet d'être dehors de l'hôpital tu sais et puis parce qu'à part de ça qu'est-ce qu'on fait à l'hôpital ? Une fois qu'on a parlé au médecin, qu'on a pris nos pilules bien on niaise, on écoute la tv puis on se berce. Moi c'est ça que je faisais t'sais. [. . . Je ne veux pas parler 
contre eux, parce qu'ils font bien leur possible à l'hôpital tu sais, mais mon dieu ça peut être déprimant des fois. (Marianne)

Bien toujours à l'hôpital t'as pas les mêmes pensées dans la tête hein ? À rester entre quatre murs, vient un temps où les ondes rebondissent sur toi-même. [. . .] Je trouve que vivre dans la communauté c'est mieux qu'être à l'hôpital, tu as plus ton intimité, tu es plus chez toi, tu es plus en confiance, si tu veux dormir tu n'es pas obligé de te lever parce que c'est l'heure du dîner. Tu sais que tu peux manger un peu plus tard en logement tu sais, puis la bouffe est meilleure qu'à l'hôpital tu sais. (Sébastien)

Bien que d'autres motifs liés au tempérament et à la personnalité doivent être pris en compte, le discours de ces participants et participantes pointe essentiellement vers la maladie et ses conséquences comme facteurs explicatifs de cette réalité. Malgré leur observance quant à la médication, les personnes ayant un mode de vie domestique sont envahies par des symptômes psychotiques qui ont pour effet de perturber leur fonctionnement social. Si dans certains cas les symptômes altèrent la vision que ces personnes ont d'ellesmêmes et de leurs capacités, et dans d'autres la vision de leur environnement social, dans tous les cas, les symptômes font obstacle à leur intégration dans la communauté.

Cela est d'autant plus vrai lorsque les personnes ne sont pas en mesure de discerner ce qui appartient ou non au monde réel. Pour ne donner qu'un exemple, le délire de persécution dans lequel est plongée Marianne fait en sorte que le monde extérieur, notamment le voisinage, constitue une menace : elle hésite donc à sortir de chez elle, de crainte d'être jugée ou encore d'être victime de représailles. D'autre part, audelà des symptômes qui peuvent être incapacitants, la conscience des pertes associées à la maladie a également un impact sur l'isolement des personnes ayant un mode domestique. En effet, très conscient de ses limites actuelles, très difficiles à accepter, et n'ayant pas envie que son entourage le voit dans cet état qu'il considère diminué, Jérémie mentionne qu'il a choisi de s'éloigner le temps de reprendre du mieux, de se rétablir. Cependant, lorsqu'il est question de l'espoir d'aller mieux, Jérémie ainsi que ses acolytes expriment des attentes et anticipations réduites.

Passant la majorité de leur temps confinées chez elles, les personnes ayant un mode domestique sont, de loin, les plus isolées lorsqu'on les compare à celles ayant un mode nomade ou social. Pourtant, parmi les 3 personnes classifiées dans cette catégorie, l'un vit avec un membre de sa famille et l'autre dans une résidence intermédiaire de groupe. En raison de leur refus de participer à des activités de groupe proposées par des organismes communautaires de leur quartier, le quotidien de ces personnes est principalement orienté autour de deux types d'occupation. D'une part, l'essentiel de leur vie tourne autour de l'accomplissement des activités de la vie quotidienne et domestique. D'autre part, s'il leur arrive à l'occasion de faire des sorties (ex : aller au restaurant, rendre visite à un proche), elles mentionnent que leurs principales occupations sont de regarder la télévision, d'écouter la radio, de lire ou encore d'écrire. Les extraits suivants donnent un aperçu de l'éventail des activités quotidiennes des personnes partageant ce mode de vie :

Bien, je me lève, je me lave, je m'habille, je fais du ménage et puis j'écoute la télé, j’écoute la radio, je regarde les journaux puis le soir je fais mes petites commissions. Tous les jours en général $\mathrm{j}$ 'ai des commissions à faire. Comme une fois c'est la banque, une autre fois c'est la pharmacie, une autre fois c'est le vidéo, une autre fois c'est l'épicerie, une autre fois c'est le centre d'achat, ça m'arrive tout le temps. (Marianne)

Moi c'est plus écrire, puis lire, puis d'écouter de la musique c'est pas mal tout. Ça m'enrichit un peu genre, ça développe ma culture un peu genre. Je fais beaucoup d'effort, j'écris beaucoup, comme j'écris peut-être 


\section{ENTRE LA PRÉSENCE ET L’INTÉGRATION SOCIALE}

une dizaine de poèmes sur dactylo, des choses comme ça genre je lis un peu genre. Je fais des activités un peu intellectuelles, mais tu sais bouger à l'extérieur, faire de l'exercice ça me demande beaucoup genre. (Jérémie)

Ainsi, à l'exception des contacts avec un ami ou une amie ou encore avec un membre de la famille, les personnes ayant un mode de vie domestique ont peu de contacts sociaux. La solitude, qu'elle soit choisie ou subie, fait partie de leur quotidien. En ce sens, les visites des professionnels et des professionnelles de l'équipe ACT sont fortement appréciées, car elles permettent d'échanger et de combler un vide ressenti.

\section{Le mode social}

Les personnes ayant un mode de vie social $(n=3)$ sont en voie d'intégration. D'ailleurs, elles en rêvent. Lorsqu'elles parlent de leurs projets d'avenir, tous incluent une composante sociale, qu'il s'agisse d'avoir un emploi rémunéré, de retourner à l'école, de se faire des amies ou des amis ou encore d'avoir une vie amoureuse. Comparativement aux personnes ayant des modes de vie nomade ou domestique, celles ayant un mode social sont définitivement des êtres d'action, des êtres proactifs. Soutenues par leur entourage et l'équipe ACT, elles travaillent fort pour que leurs rêves deviennent réalité. En fait, ce sont des personnes qui évoluent, qui se transforment et qui ont atteint un certain niveau de bien-être :

J'ai du temps. Ça fait que là, je le savoure comme le moment présent là. Je savoure, tu sais, là je suis bien, je ne suis pas stressée. J'ai ma petite routine. Puis comme ça rien de dérangeant. Je trouve que je mène une bonne vie en ce moment. Puis j'veux dire, ça ne fait pas si longtemps que je trouve ça là tu sais. Tu sais ça fait tellement longtemps que je ne me sens pas bien. Que profiter que je me sente bien, tu sais que je goûte à ça là. Fait que je trouve important de prendre le temps. Puis après ça, je vais être mieux pour me lancer dans quoi que ce soit. (Rose)

Il y a eu beaucoup, plusieurs changements dans ma vie. Présentement, pour moi, c'est une autre étape de ma vie la. C'est comme si j'avais une seconde chance là. Puis, comment je vois ca ? Bien je veux voir ça le plus beau possible là. Je ne veux pas d'ombre dans ma nouvelle vie là. [Qu'est-ce qui a changé ?] C'est le système de valeurs qui a changé. La maintenant, j'ai de la volonté, j'ai arrêté de fumer. Ensuite, j’ai de la volonté, j'ai accepté les choses que je ne peux pas changer. Ça va changer avec le temps tu sais. Moi j'appelle ça la période de la sérénité. J'ai pris de la gratitude, j'ai pris du courage. (Élisabeth)

Les personnes ayant un mode social considèrent avoir trouvé un certain équilibre dans leur vie. S'agit-il de l'œuvre du temps, du bon niveau de fonctionnement pré-morbide, de la reconnaissance et de l'acceptation de la maladie et de ses conséquences, de l'efficacité du traitement pharmacologique et psychosocial, ou encore de stratégies plus adaptées pour faire face aux difficultés rencontrées ? Quoi qu'il en soit, ces facteurs jouent certainement sur leur niveau de bien-être actuel. Si la maladie est encore présente, se manifestant surtout par des symptômes de type anxieux, l'absence de symptômes psychotiques facilite certainement leur parcours d'insertion dans la communauté.

Même s'il n'en a pas toujours été ainsi, les personnes ayant un mode de vie social ont su acquérir, au fil du temps et des expériences, l'autonomie nécessaire à la vie en appartement. À l'exception de Denis, un participant qui en partage la responsabilité avec ses colocataires, ces personnes assument seules l'ensemble des tâches domestiques qui incombent à la vie en appartement. Il importe par ailleurs de souligner que comparativement aux personnes ayant un mode domestique, ces tâches ne constituent qu'une partie de la vie de celles ayant un mode social. Plutôt, ces dernières recherchent le contact humain et souhaitent contribuer 
à la société ; c'est pourquoi elles sont engagées dans des activités de nature socioprofessionnelle à raison de 2-3 jours par semaine. Tout en donnant une structure à leur vie, le travail, indiquent-elles, constitue une importante source de développement et de valorisation personnelle. Il importe cependant de souligner que pour elles, cet engagement dans un travail bénévole ou offrant une modeste compensation financière est vu comme une étape, un tremplin vers l'obtention d'un emploi rémunéré. Les propos de Rose sont assez évocateurs à cet égard.

Des fois, je me dis ce serait idéal de se faire rémunérer. Mais après ça, tu sais veux dire, tu t’y fais. Tu te dis que ça va arriver d'avoir un emploi rémunéré. Tu sais je suis active. Ce n'est pas comme si ça faisait deux ans et demi que je ne faisais rien là. Tu sais je veux dire je suis active, je suis dans mon domaine aussi, ça fait que ça ne fait pas de trou dans mon CV. Fait que je me dis ça va venir, mais là au moins là j'ai le temps de m'occuper de moi. Ça fait que c'est très important. Bien ça me garde occupée, ça me garde active. Tu sais ce que je veux dire. Je ne suis pas là à essayer de remplir des trous dans ma journée, puis de dormir jusqu'à $10 \mathrm{~h}-11 \mathrm{~h}$ le matin. Tu sais que j'ai une vie plus équilibré, plus saine. (Rose)

Outre ces activités de nature socioprofessionnelle, les personnes ayant un mode social participent également à des activités de loisirs, qu'elles réalisent seules, avec des amis ou amies ou encore avec des membres de leurs familles (ex : aller voir un match d'improvisation, un concert, une pièce de théâtre). Elles participent assidûment aux activités proposées par l'équipe ACT, mais évoluent également en dehors des services proposés dans le système de santé mentale, participant notamment aux activités initiées dans leur milieu de travail. Cependant, leurs revenus limités les empêchent d'accéder à un certain nombre d'activités auxquelles elles souhaiteraient participer. En outre, moins isolées que les autres, ces personnes ont des liens significatifs avec les membres de leur famille. Celles-ci constituent leur principale source de soutien car leur réseau d'amis et d'amies demeure limité. Se sentant parfois seules, elles apprécient les visites et les échanges qu'elles ont avec les intervenants et intervenantes de l'équipe ACT. Ainsi, l'équipe a une place importante dans leur vie, les aidant à cheminer dans la communauté.

\section{DISCUSSION}

Les résultats de cette étude doivent être interprétés à la lumière de certaines limites méthodologiques et biais, tels que la petite taille de l'échantillon, les critères d'inclusion de l'échantillon (ex : nécessité d'avoir passé plus de $75 \%$ de temps dans la communauté), l'utilisation d'une méthode d'échantillonnage non probabiliste (pouvant conduire à des biais de sélection) et le fait que le recrutement ait été effectué au sein d'une seule équipe ACT. Ils doivent aussi être lus en tenant compte du fait que les personnes rencontrées sont celles dont les troubles mentaux sont les plus graves et dont l'adaptation, puis l'intégration sociale, ont toujours représenté un défi. Ainsi, une recherche menée auprès d'individus ayant des troubles moins graves aurait peut-être donné lieu à l'identification d'autres modes de vie que ceux répertoriés ici. Bien que nos données ne puissent être généralisées, elles éclairent sur un certain nombre d'enjeux associés à la pratique du suivi intensif en équipe dans la communauté. En effet, ces connaissances à l'égard des modes de vie des personnes intégrées à une équipe ACT lèvent le voile sur des avenues d'intervention à privilégier.

Le premier constat qui émerge des données recueillies est à l'égard de la diversité d'individus recevant des services d'une équipe ACT. Alors que l'exhaustivité et la spécificité des critères d'admission à ces équipes pourrait laisser croire à une certaine homogénéisation de la clientèle, on a retrouvé au sein de cet échantillon 
des individus qui se distinguaient au plan de leur façon d'être et d'interagir dans la communauté, rejoignant en cela les propos de Harding et Zahniser (1994) sur l'hétérogénéité de la population atteinte de schizophrénie. Ainsi, en dépit de leur similitude au plan du diagnostic, de leur histoire d'hospitalisations, de leur isolement social et de leur précarité économique, les personnes rencontrées n'ont pas le même rapport à la communauté. Ce constat appuie l'importance de certains principes d'intervention mis de l'avant par les défenseurs du modèle ACT, notamment l'intervention complète, individualisée et interdisciplinaire (Allness \& Knoedler, 1998 ; Stein \& Santos, 1998). Qui plus est, ces résultats suggèrent l'importance de l'intervention intégrée, notamment pour répondre aux besoins des personnes ayant un mode de vie nomade en matière d'alcoolisme et de toxicomanie, et au désir d'intégration de celles ayant un mode de vie social. Ainsi, l'embauche d'un intervenant ou d'une intervenante ayant une spécialisation en toxicomanie, de même qu'un agent ou qu'une agente d'intégration socioprofessionnelle, tel que proposé dans les normes d'implantation et de fidélité des équipes ACT, s'avère un incontournable (Association des hôpitaux du Québec, 2004).

Le deuxième constat qui émerge de cette étude est lié à la motivation au changement. Quoi que plus manifeste chez les personnes ayant un mode de vie nomade, l'ambivalence au changement a été observée chez l'ensemble des participants et des participantes. Selon les travaux de Rogers (1975), celle-ci serait liée à l'interaction entre deux variables : (a) le malaise ressenti et (b) l'espoir de la personne en sa capacité de résoudre les difficultés rencontrées. Sans nier l'interaction entre ces deux variables, les données que nous avons recueillies suggèrent plutôt que l'ambivalence des personnes ayant un mode nomade serait d'abord et avant tout due à l'absence de reconnaissance des difficultés et, en l'occurrence, de malaise face à la situation, alors que celle des personnes ayant un mode domestique ou social parait surtout liée à un faible niveau d'espoir. Ce niveau d'espoir serait intimement lié au sentiment de compétence personnelle et aux capacités d'adaptation, de même qu'à l'équilibre psychologique de l'individu (J.F. Miller, 1992).

À cet égard, l'utilisation de stratégies pour instiguer l'espoir par les professionnels et professionnelles des équipes ACT parait être une avenue d'intervention devant être privilégiée (Russinova, 1999). Cela est d'autant plus important que ces stratégies permettent également de consolider le lien unissant la personne à l'équipe ACT (Chinman et al., 1999). Ainsi, considérant la relation de confiance comme un ingrédient essentiel au succès de l'intervention, les intervenants et intervenantes auraient tout avantage à user de ces stratégies auprès des personnes ayant un mode de vie nomade qui gardent les professionnels et professisonnelles à distance et s'en méfient (Frank \& Gunderson, 1990). D'autre part, notamment afin de travailler à l'accroissement du malaise face à la situation problème, inconfort nécessaire au changement, l'utilisation de l'entretien motivationnel constituent des avenue prometteuses (W.R. Miller \& Rollnick, 2002). Parce qu'elle consiste directement à travailler sur l'ambivalence de la personne, notamment en soulevant les avantages et inconvénients associés au changement, cette approche pourrait faciliter le travail vers l'atteinte d'un plus grand niveau d'intégration.

Enfin, le dernier constat qui émerge ici est lié à l'isolement social des personnes intégrées à une équipe $\mathrm{ACT}$. Bien qu'à des degrés variables, toutes les personnes rencontrées dans cet échantillon souffraient de solitude. Cette réalité nous conduit à soulever l'un des risques inhérents à la pratique du suivi intensif : celui de maintenir la personne dans son isolement actuel ou encore d'aggraver la situation. D'une part, l'intervention à domicile peut contribuer au maintien de l'isolement. Cela est d'autant plus vrai pour les personnes ayant un mode domestique, qui ne quittent que très rarement leur milieu de vie et qui présentent une tendance à 
la passivité : ainsi, le fait que les intervenants et intervenantes se déplacent à la maison ne constitue pas un incitatif à aller vers la communauté. Cependant, ce piège peut facilement être contourné par la diversification des lieux de rencontre avec l'équipe ACT, lieux qui seront minutieusement sélectionnés pour répondre aux objectifs visés par l'intervention.

D'autre part, l'intégration à une équipe $\mathrm{ACT}$ peut conduire à un éloignement ou à une diminution du soutien apporté par les proches. L'approche d'équipe, l'intervention complète, l'intensité des services sont au nombre des facteurs en cause dans ce processus. Les intervenantes et intervenants des équipes ACT doivent être conscients de ce risque et doivent se garder de remplacer les solidarités naturelles. En ce sens, l'intervention familiale parait être une avenue à privilégier, car elle permet d'outiller les proches et ainsi de diminuer les probabilités d'épuisement et d'éloignement (McFarlane, Statsny, \& Deakins, 1992). Qui plus est, et cela est attesté par nombre d'études, l'intervention familiale aurait un effet protecteur en ce qui concerne les rechutes associées à un trouble psychotique (Pitschel-Walz, Leucht, Bäuml, Kissling, \& Engel, 2001).

Au-delà des risques encourus et en tenant compte des avenues d'intervention à privilégier pour y faire face, des moyens doivent être mis en œuvre pour faire face à la solitude des personnes intégrées à une équipe $\mathrm{ACT}$. Si, tel que mentionné précédemment, l'intégration à l'emploi facilite le développement de liens sociaux, elle ne doit pas être envisagée comme l'unique solution. Ainsi, nos résultats indiquent que le travail n'est pas un objectif prioritaire pour tous : si les personnes ayant un mode social souhaitent obtenir un emploi rémunéré, les personnes ayant un mode nomade ou domestique ne partagent pas ce rêve. C'est pourquoi le développement de mesures alternatives, telles que le soutien à la socialisation, est une avenue qui devrait être privilégiée au sein des équipes ACT (Davidson, Haglund et al., 2001 ; Davidson et al., 2004). En somme, dans une perspective d'intervention centrée sur le rétablissement, différentes mesures de soutien devraient être proposées dans l'optique de faciliter l'intégration sociale des personnes ayant des TMG et ce, en tenant compte de leur mode de vie.

\section{CONCLUSION}

Les résultats présentés dans cet article ont permis de jeter un regard sur le cheminement de 9 personnes intégrées à une équipe de type ACT. De façon plus spécifique, les données issues de cette étude ont démontré qu'au-delà de certaines caractéristiques communes, les personnes qui font partie de ce type de programme ont des expériences de vie communautaire fort diversifiées. Ces expériences sont teintées par leurs caractéristiques individuelles et par leurs interactions avec l'environnement. S'il convient mieux, dans le cas des personnes ayant un mode de vie nomade ou domestique, de parler de maintien ou de présence dans la communauté, celles ayant un mode social sont définitivement engagées dans une trajectoire qui les mènera peut-être à réaliser leur rêve : celui d'être des citoyens et citoyennes « comme tout le monde » : c'est-à-dire qui ont pu développer des amitiés, qui sont engagées dans une relation amoureuse, qui ont un travail rémunéré et qui ont des activités sociales significatives.

Nos résultats, qui favorisent une meilleure compréhension des modes de vie des personnes intégrées à des équipes de type $\mathrm{ACT}$, permettent également l'amorce d'une réflexion à l'égard des composantes d'intervention essentielles à l'atteinte d'un plus haut niveau d'intégration dans la communauté. Au-delà des personnes intégrées à une équipe $\mathrm{ACT}$, il serait intéressant d'étendre la réflexion sur les modes de vie à 


\title{
ENTRE LA PRÉSENCE ET L’INTÉGRATION SOCIALE
}

d'autres groupes d'individus ayant des troubles mentaux et recevant des services dans la communauté. Le tout, afin de confirmer si les tendances observées ici se reproduisent chez d'autres groupes et, s'il y a lieu, de contribuer à la construction d'une typologie liée au fonctionnement et à l'intégration sociale des personnes ayant des troubles mentaux graves.

\section{NOTES}

1. Développé aux États-Unis, le modèle est mieux connu sous le nom d'Assertive Community Treatment. En accord avec le guide de pratique développé par l'Association des hôpitaux du Québec (2004), l'acronyme $A C T$ sera utilisé tout au nom de l'article.

2. Cette étude doctorale, menée au Centre hospitalier Robert-Giffard (actuellement nommé l'Institut universitaire en santé mentale de Québec) s'est étendue sur une période de 5 ans, soit de 2004 à 2009. Cependant, la période de collecte de données s'est déroulée sur une période d'un an, soit du mois d'avril 2004 au mois de mars 2005.

3. Le terme mode de vie (en anglais lifestyle) a été privilégié ici en référence à sa définition sociologique, c'est-à-dire la façon d'être, d'interagir, de se divertir, de penser d'un individu.

\begin{abstract}
Forty years after the beginning of deinstitutionalization, social integration still remains an issue among people who have serious mental illnesses. In fact, their living conditions are marked by social as well as economic exclusion. In response to this situation and to support people in their journey toward social integration, the Program of Assertive Community Treatment (PACT) has been developed. While until now the focus of PACT research has been primarily evaluative, this exploratory qualitative study was undertaken to identify the factors which help or hinder social integration. The analysis of clinical records and semistructured interviews revealed a diversity of community experiences among the 9 participants in this study. Indeed, the within-case and cross-case analysis made it possible to highlight 3 types of lifestyle (nomadic, domestic, and social), reflecting the ways these individuals interact and participate in society. Our results raise several concerns about the practice of Assertive Community Treatment.
\end{abstract}

Keywords: Social integration, social exclusion, lifestyles, community life, serious mental illnesses, schizophrenia, Assertive Community Treatment

\section{RÉFÉRENCES}

Allness, D.J., \& Knoedler, W.H. (1998). The PACT model of community-based treatment for persons with severe and persistent illnesses: A manual for PACT start-up. Arlington, VA : National Alliance on Mental Illness.

Allott, P., Loganathan, L., \& Fulford, K.W.M. (2002). Discovering hope for recovery from a British perspective: A review of a selection of recovery literature, implications for practice and systems change. Canadian Journal of Community Mental Health, 21(3), 13-33.

Angell, B. (2003). Contexts of social relationship development among Assertive Community Treatment clients. Mental Health Services Research, 5(1), 13-25.

Anthony, W.A. (2004). The principle of personhood: The field's transcendent principle. Psychiatric Rehabilitation Journal, 27(3), 205.

Anthony, W.A., \& Blanch, A. (1987) Supported employment for persons who are psychiatrically disabled: An historical and conceptual perspective. Psychosocial Rehabilitation Journal, 11, 5-23.

Association des hôpitaux du Québec. (2004). Guide pratique pour les équipes de suivi intensif dans la communauté. Montréal, QC : Auteur.

Aubry, T., \& Myner, J. (1996). Community integration and quality of life: A comparison of persons with psychiatric disabilities in housing programs and community residents who are neighbours. Canadian Journal of Community Mental Health, 15(1), 5-20. 
Aviram, U., \& Segal, S.P. (1977). The exclusion of the mentally ill: Reflexion on an old problem in a new context. Archives of General Psychiatry, 29, 126-131.

Bond, G.R., Salyers, M.P., Rollins, A.L., Rapp, C.A., \& Zipple, A.M. (2004). How evidence-based practices contribute to community integration. Community Mental Health Journal, 40(6), 569-588.

Bradshaw, W., Armour, M.P., \& Roseborough, D. (2007). Finding a place in the world: The experience of recovery from severe mental illness. Qualitative Social Work, 6(1), 27-47.

Chinman, M., Allende, M., Bailey, P., Maust, J., \& Davidson, L. (1999). Therapeutic agents of Assertive Community Treatment. Psychiatric Quarterly, 70(2), 137-162.

Cohen, C.I., \& Sokolovsky, J. (1978). Schizophrenia and social networks: Ex-patients in the inner city. Schizophrenia Bulletin, 4(4), 546-550.

Colbert, P., Dubois, L., Fleury, C., \& Giguère, P.-A. (2003). Programme clinique : suivi intensif en équipe dans la communauté. Québec, QC : Centre hospitalier Robert-Giffard.

Cormier, C. (2009). L'espoir d'un mieux-être malgré la schizophrénie : témoignages de personnes utilisatrices de services vivant dans la communauté. Québec, QC : Université Laval.

Dailey, W.F., Chinman, M.J., Davidson, L., Garner, L., Vavrousek-Jakuba, E., Essock, S., . . Tebes, J.K. (2000). How are we doing?: A statewide survey of community adjustment among people with serious mental illness receiving intensive outpatient services. Community Mental Health Journal, 36(4), 363-382.

Davidson, L., Haglund, K.E., Stayner, D.A., Rakfeldt, J., Chinman, M. J., \& Tebes, J.K. (2001). "It was just realizing ... that life isn't one big horror": A qualitative study of supported socialization. Psychiatric Rehabilitation Journal, 24(3), 275-292.

Davidson, L., Hoge, M., Godleski, L., Rakfeldt, J., \& Griffith, E. (1996). Hospital or community living?: Examining consumer perspectives on deinstitutionalization. Psychiatric Rehabilitation Journal, 19, 49-58.

Davidson, L., Shahar, G., Stayner, D.A., Chinman, M.J., Rakfeldt, J., \& Tebes, J.K. (2004). Supported socialization for people with psychiatric disabilities: Lessons from a randomized controlled trial. Journal of Community Psychology, 32(4), 453-477.

Davidson, L., Stayner, D., Nickou, C., Styron, T.H., Rowe, M., \& Chinman, M.L. (2001). Simply to be let in: Inclusion as a basis for recovery. Psychiatric Rehabilitation Journal, 24(4), 375-388.

Drake, R.E. (1998). Brief history, current status, and future place of Assertive Community Treatment. American Journal of Orthopsychiatry, 68(2), 172-175.

Frank, A., \& Gunderson, J.G. (1990). The role of the therapeutic alliance in the treatment of schizophrenia. Archives of General Psychiatry, 47, 228-236.

Gélinas, D. (2002). Le suivi intensif en équipe dans la communauté et la réadaptation psychosociale font-ils bon ménage ? Dans Suivi intensif en équipe dans la communauté : fidélité au modèle ACT et stratégie d'implantation. Colloque de l'Association des hôpitaux du Québec, Montréal, QC.

Granerud, A., \& Severinsson, E. (2006). The struggle for social integration in the community: The experiences of people with mental health problems. Journal of Psychiatric and Mental Health Nursing, 13(3), 288-293.

Gulcur, L., Tsemberis, S., Stefancic, A., \& Greenwood, R.M. (2007). Community integration of adults with psychiatric disabilities and histories of homelessness. Community Mental Health Journal, 43(3), 211-228.

Guy, S. (1997). Assertive Community Treatment of the long-term mentally ill. Journal of the American Psychiatric Nurses Association, 3(6), 185-190.

Harding, C.M., \& Zahniser, J.H. (1994). Empirical correction of seven myths about schizophrenia with implications for treatment. Acta Psychiatrica Scandinavica, 90(Supp. 384), 140-146.

Heider, D., Angermeyer, M.C., Winkler, I., Schomerus, G., Bebbington, P.E., Brugha, T., . . Toumi, M. (2007). A prospective study of quality of life in schizophrenia in three European countries. Schizophrenia Research, 93, 194-202.

Kennedy, C. (1989). Community integration and well-being: Toward the goal of community care. Journal of Social Issues, 45(3), 65-77.

Krupa, T., Eastabrook, S., Hern, L., Lee, D., North, R., Percy, K., . . Wing, G. (2005). How do people who receive Assertive Community Treatment experience this service? Psychiatric Rehabilitation Journal, 29(1), 18-24.

Kruzich, J.M. (1985). Community integration of the mentally ill in residential facilities. American Journal of Community Psychology, 13(5), 553-564. 


\section{ENTRE LA PRÉSENCE ET L'INTÉGRATION SOCIALE}

Latimer, E.A. (1999). Economic impacts of Assertive Community Treatment: A review of the literature. Canadian Journal of Psychiatry, 44, 443-454.

Leff, J., \& Warner, R. (2006). Social inclusion of people with mental illness. Cambridge, Angleterre : Cambridge University Press.

Lemaire, G.S., \& Mallik, K. (2005). Barriers to community integration for participants in community-based psychiatric rehabilitation. Archives of Psychiatric Nursing, 19(3), 125-132.

McFarlane, W.R., Stastny, P., \& Deakins, S. (1992). Family-aided Assertive Community Treatment: A comprehensive rehabilitation and intensive case management approach for persons with schizophrenic disorders. New Direction for Mental Health Services, 53, 43-54.

McGrew, J.H., Wilson, R.G., \& Bond, G.R. (1996). Client perspective on helpful ingredients of Assertive Community Treatment. Psychiatric Rehabilitation Journal, 19(3), 13-21.

Miles, M.B., \& Huberman, A.M. (2003). Analyse des données qualitatives ( $2^{\mathrm{e}}$ éd). Paris, France : De Boeck.

Miller, J.F. (1992). Inspiring hope. Dans J.F. Miller, Coping with chronic illness: Overcoming powerlessness (pp. 413433). Philadelphia, PA : F.A. Davis.

Miller, W.L., \& Crabtree, B.F. (1999). The dance of interpretation. Dans B.F. Crabtree \& W.L. Miller (dir.), Doing qualitative research (pp. 127-143). Thousand Oaks, CA : Sage.

Miller, W.R., \& Rollnick, S. (2002). Motivational interviewing: Preparing people for change ( $2^{\mathrm{e}}$ éd.). New York, NY : Guilford.

Moldovan, V. (2007). Attitudes of mental health workers toward community integration of the persons with serious and persistent mental illness. American Journal of Psychiatric Rehabilitation, 10(1), 19-30.

Nagy, M.P., Fisher, G.A., \& Tessler, R.C. (1988). Effects of facility characteristics on the social adjustment of mentally ill residents of board-and-care homes. Hospital and Community Psychiatry, 39, 1281-1286.

Nordt, C., Müller, B., Rössler, W., \& Lauber, C. (2007). Predictors and course of vocational status, income, and quality of life in people with severe mental illness: A naturalistic study. Social Science and Medicine, 65(7), 1420-1429.

Onken, S.J., Craig, C.M., Ridgway, P., Ralph, R.O., \& Cook, J.A. (2007). An analysis of the definitions and elements of recovery: A review of the literature. Psychiatric Rehabilitation Journal, 31(1), 9-22.

Patton, M.Q. (1990). Qualitative evaluation and research methods. Thousand Oaks, CA : Sage.

Pitschel-Walz, G., Leucht, S., Bäuml, J., Kissling, W., \& Engel, R.R. (2001). The effect of family interventions on relapse and rehospitalisation in schizophrenia: A meta-analysis. Schizophrenia Bulletin, 27(1), 73-92.

Prince, P.N., Demidenko, N., \& Gerber, G.J. (2000). Client and staff members' perceptions of Assertive Community Treatment: The nominal groups technique. Psychiatric Rehabilitation Journal, 23(3), 285-288.

Prince, P.N., \& Prince, C.R. (2002). Perceived stigma and community integration among clients of Assertive Community Treatment. Psychiatric Rehabilitation Journal, 25(4), 323-331.

Roe, D. (2001). Progressing from patienthood to personhood across the multidimensional outcomes in schizophrenia and related disorders. Journal of Nervous and Mental Disease, 189(10), 691-699.

Rogers, R.W. (1975). A protection motivation theory of fear appeals and attitude change. Journal of Psychology, 91(1), 93-114.

Rosenblatt, A., \& Mayer, J.E. (1974). The recidivism of mental patients: A review of past studies. American Journal of Orthopsychiatry, 44(5), 697-706.

Russinova, Z. (1999). Providers' hope-inspiring competence as a factor optimizing psychiatric rehabilitation outcomes. Journal of Psychiatric Rehabilitation, 65(4), 50-57.

Sayce, L., \& Measey, L. (1999). Strategies to reduce social exclusion for people with mental health problems. Psychiatric Bulletin, 23, 65-67.

Segal, S.P., \& Everett-Dille, L. (1980). Coping styles and factors in male/female social integration. Acta Psychiatrica Scandinavica, 60, 8-20.

Stein, L.I., \& Santos, A.B. (1998). Assertive Community Treatment of persons with severe mental illness. New York, NY : W.W. Norton.

Stein, L.I., \& Test, M.A. (1980). An alternative to mental hospital treatment. I. Conceptual model, treatment program, and clinical evaluation. Archives of General Psychiatry, 37, 392-397.

van Wel, T., Felling, A., \& Persoon, J. (2003). The effect of psychiatric rehabilitation on the activity and participation level of clients with long-term psychiatric disabilities. Community Mental Health Journal, 39(6), 535-546. 
Ware, N.C., Hopper, K., Tugenberg, T., Dickey, B., \& Fisher, D. (2007). Connectedness and citizenship: Redefining social integration. Psychiatric Services, 58(4), 469-474.

Wieland, M.E., Rosenstock, J., Kelsey, S., Ganguli, M., \& Wisniewski, S.R. (2007). Distal support and community living among individuals diagnosed with schizophrenia and schizoaffective disorder. Psychiatry, 70(1), 1-11. 